\title{
Chagas cardiomyopathy associated with serological cure after trypanocidal treatment during childhood
}

\author{
Marisa Liliana Fernández ${ }^{[1]}$, Yolanda Hernández ${ }^{[1]}, K_{\text {Karenina Scollo }}^{[2]}$, \\ Mónica Ines Esteva ${ }^{[3]}$, Adelina Rosa Riarte ${ }^{[1]}$ and Nilda Graciela Prado ${ }^{[1]}$
}

[1]. Departamento de Clínica, Patologia y Tratamiento, Instituto Nacional de Parasitología Dr. Mario Fatala Chaben, Ministerio de Salud, Buenos Aires, Argentina. [2]. Departamento de Diagnostico, Instituto Nacional de Parasitología Dr. Mario Fatala Chaben, Ministerio de Salud, Buenos Aires, Argentina.

[3]. Departamento de Investigación, Instituto Nacional de Parasitología Dr. Mario Fatala Chaben, Ministerio de Salud, Buenos Aires, Argentina.

\begin{abstract}
Chagas disease is a chronic parasitological disease, which could cause cardiac manifestations in approximately one-third of affected individuals. Benznidazole and nifurtimox are used to treat this parasitological infection caused by Trypanosoma cruzi. Conventionally, the criterion for cure is consistently negative serological tests after treatment. We report a case of a patient who was treated when she was 13 years old and achieved T. cruzi negative seroconversion but developed Chagas disease cardiomyopathy as an adult.
\end{abstract}

Keywords: Chagas disease treatment. Negative seroconversion. Cardiomyopathy.

\section{INTRODUCTION}

Chagas disease is a chronic parasitological disease, which could cause cardiac manifestations in adulthood. Two randomized clinical trials and other studies have shown that treatment of Chagas disease in children using benznidazole is considered to be highly effective owing to the high rate of negative seroconversion ${ }^{1,2}$.

\section{CASE REPORT}

A 52-year-old woman was diagnosed with Chagas disease when she was 13 years old. She was treated with pills administered twice daily for 3 months. During her childhood, the patient lived in Ingenio La Esperanza (a sugar mill colony) located in the province of Jujuy, which at that time was an endemic area for Chagas disease. At the age of 14 years, she moved to Buenos Aires (the capital of Argentina), a city free of the Trypanosoma cruzi vector. When she was shown a bottle of Radanil ${ }^{\circledR}$ (benznidazole - Roche Lab Argentina), she immediately recognized it and explained that she remembered it easily because as a child she was punished if she did not take the pills. Her serology against $T$. cruzi was non-reactive on several occasions in her young adulthood; thus, she was told that she was cured.

Corresponding author: Marisa L Fernandez.

e-mail: marisa.fernandez@gmail.com

Received 21 October 2017

Accepted 8 May 2018
Upon presentation to our Institute, her non-reactive serology was confirmed twice using enzyme-linked immunosorbent assay (ELISA), immunofluorescent antibody tests, and indirect hemagglutination assay. Additionally, the polymerase chain reaction test for detection of $T$. cruzi was undetectable twice. An electrocardiogram showed a complete right bundle block and a left anterior fascicular block, and 24-hour Holter showed sinus rhythm with a mean cardiac rate of 73 beats $/ \mathrm{min}$ and 492 ventricular premature beats without any ventricular tachycardia or significant pauses. An echocardiogram showed normal parameters. We ruled out other etiologies including congenital cardiomyopathy, arterial hypertension, or the consumption of alcohol and/or other cardiotoxic substances, which could be associated with this condition. These results in an individual diagnosed with chronic $T$. cruzi infection are categorized as Chagas cardiomyopathy Stage I based on the Kuschnir classification ${ }^{3}$. When informed of this diagnosis, the patient could not understand how she could have developed Chagas cardiomyopathy despite being declared cured.

\section{DISCUSSION}

Chagas disease is a chronic parasitological disease, which can cause cardiac manifestations in approximately one-third of affected individuals. Chagas' myocarditis is the most common type of non-ischemic cardiomyopathy in Latin America. It is usually observed in adulthood, several decades after $T$. cruzi infection. Chagas cardiomyopathy is commonly diagnosed based on asymptomatic conduction disturbances typical of Chagas disease and severe forms of disease including heart failure, arrhythmias, thromboembolic 
events, and eventually sudden death ${ }^{4}$. Electrocardiographic abnormalities considered highly indicative but not specific for Chagas disease are right bundle branch block with or without associated left anterior fascicular block, $2^{\text {nd }}$ - and $3^{\text {rd }}$-degree atrioventricular blocks, sinus bradycardia at a rate $<50$ beats/ min, sinus node dysfunction, left bundle branch block, and atrial fibrillation ${ }^{5}$. Notably, 24-hour Holter findings of frequent ventricular premature beats and polymorphous or repetitive non-sustained ventricular tachycardia are associated with T. cruzi etiology ${ }^{6}$. Echocardiographic findings include structural myocardial disturbances manifested as segmental wall motion abnormalities, apical left ventricular aneurysm, left ventricular systolic dysfunction, and dilatation of cardiac cavities. Our patient showed a complete right bundle branch block, a left anterior fascicular block, and ventricular premature beats, which are strongly indicative of but not specific for Chagas cardiomyopathy, without echocardiographic abnormalities. Treatment options for T. cruzi infection are benznidazole and nifurtimox. Both drugs have been used since their discovery in 1967 and 1976, respectively ${ }^{7}$. Conventionally, the criterion for cure is sustained negative serological tests after treatment. This negative seroconversion implies the clearance of parasites, which act as a stimulus for a chronic immune response, which triggers the production of specific antibodies. Experts in the management of Chagas disease have established that cure in patients with acute $T$. cruzi infection should be followed by consistently negative serological tests 12 months after infection, and in chronic infection, decades of follow-up are required to prove this negative seroconversion ${ }^{8}$.

ELISA, immunofluorescent antibody tests, and indirect hemagglutination assay are the most commonly used tests for Chagas disease. Serodiagnostic confirmation of T. cruzi infection requires at least two reactive tests because no assay shows sufficient sensitivity and specificity to be used alone 9 . Data suggest that the sensitivity of serological assays varies based on geographical location, possibly because of differences in $T$. cruzi strains and the resulting antibody responses ${ }^{10}$, and occasionally false-negative results have been demonstrated in severely immunocompromised patients with human immunodeficiency virus infection/acquired immune deficiency syndrome. Several studies have shown marked variability in the results obtained with both drugs, although they do share a few characteristics including better performance in serological response during the acute phase and in children. Two doubleblind randomized clinical trials have studied this condition in children. In the first trial performed in the State of Goiás (Brazil), treatment with benznidazole was effective in 55.8\% of the cases with a significant decrease in serological titers after three years ${ }^{1}$. The second clinical trial using a similar protocol was reported in Salta (Argentina). Negative seroconversion was observed in $62 \%$ of the benznidazole-treated group but not in the control group ${ }^{2}$. Pharmacological treatment was universally recommended for children after similar results were observed in two randomized clinical trials. Over the last few years, two prospective randomized clinical trials performed in adults studied clinical outcomes and observed that treatment with benznidazole was ineffective in preventing clinical progression.
One of the trials, a multicenter study named BENEFIT was performed in Brazil, Colombia, El Salvador, Bolivia and Argentina. The researchers concluded that patients with Chagas cardiomyopathy treated with benznidazole and followed over 5 years showed a reduction in parasite burden but the drug did not significantly reduce clinical worsening of cardiac function and mortality. The other trial, a single-center study named TRAENA was performed in Buenos Aires, Argentina. The researchers concluded that benznidazole reduced conventional ELISA reactivity by $29 \%$ after 10 years of follow up but did not reduce the morbidity and mortality of participants (most participants were without cardiomyopathy $)^{11}$.

Another report, similar to our case report, has recently been published. A 27-year-old man with chronic Chagas disease without cardiomyopathy was treated with benznidazole and after 30 years of follow-up demonstrated the criterion for cure with negative seroconversion. However, he developed Chagas cardiomyopathy stage III based on Kuschnir classification and eventually died of this cardiomyopathy ${ }^{12}$.

In conclusion, in patients with Chagas disease, it is extremely difficult to determine whether a particular intervention would be successful in reducing morbi-mortality because it is expected that only one-third of $T$. cruzi-infected patients are likely to develop clinical (cardiac) manifestations after decades of infection. Historically, the classical criterion for cure was based on sustained negative seroconversion. Unfortunately, our patient showed negative seroconversion after trypanocidal treatment in her childhood but developed electrocardiographic changes suggesting Chagas cardiomyopathy in her adulthood.

This case report will alert the medical and scientific community regarding this condition. We recommend lifelong clinical monitoring in treated as well as untreated patients with a history of $T$. cruzi infection, regardless of their reactive or non-reactive serological status after treatment. Early diagnosis of severe cardiomyopathy and prompt treatment improves the patient's quality of life and could reduce the risk of sudden death. There remains an important knowledge gap regarding the pathogenesis of cardiomyopathy in Chagas disease. The classic criterion of cure should be reassessed, and further discussion is warranted. Surrogate markers are needed to screen patients at risk of developing cardiomyopathy and to determine the interventions that improve, inhibit, and reduce the development of this condition.

\section{Conflict of interest}

The authors declare that there is no conflict of interest.

\section{Acknowledgments}

We thanks to Marina Feraud and Laura Lafuente for assistance with improving the language of this manuscript.

\section{Financial support}

This study was funded by Administración Nacional de Laboratorios e Institutos de Salud, ANLIS, Dr CG Malbran, Ministerio de Salud de la Nación, Argentina. 


\section{REFERENCES}

1. de Andrade AL, Zicker F, de Oliveira RM, Almeida S, Luquetti A, Travassos LR, et al. Randomised trial of efficacy of benznidazole in treatment of early Trypanosoma cruzi infection. Lancet. 1996;348(9039):1407-13.

2. Sosa Estani S, Segura EL, Ruiz AM, Velázquez E, Porcel BM, Yampotis C. Efficacy of chemotherapy with benznidazole in children in the indeterminate phase of Chagas' disease. Am J Trop Med Hyg. 1998;59(4):526-9

3. Kuschnir E, Sgammini H, Castro R, Evequoz C, Ledesma R, Brunetto J. Evaluation of cardiac function by radioisotopic angiography, in patients with chronic Chagas cardiopathy. Arq Bras Cardiol. 1985;45(4):249-56.

4. Ribeiro AL, Nunes MP, Teixeira MM, Rocha MO. Diagnosis and management of Chagas disease and cardiomyopathy. Nat Rev Cardiol. 2012;9(10):576-89.

5. Prata A. Clinical and epidemiological aspects of Chagas disease. Lancet Infect Dis. 2001;1:92-100.

6. Biolo A, Ribeiro A, Clausell N. Chagas Cardiomyopathy Where do we stand after a hundred years? Prog Cardiovasc Dis. 2010;52(4):300-16.
7. Cançado JR. Terapêutica específica. In: Dias JCP, Coura JR, editors. Clínica e terapêutica da doença de Chagas: uma abordagem prática para o clínico geral. $1^{\text {st }}$ edition. Rio de Janeiro: Editorial FIOCRUZ; 1997. p. 323-51.

8. Cançado JR. Long term evaluation of etiological treatment of Chagas disease with benznidazole. Rev Inst Med Trop Sao Paulo. 2002;44(1):29-37.

9. Araújo $\mathrm{AB}$, Berne ME. Conventional serological performance in diagnosis of Chagas' disease in southern Brazil. Braz J Infect Dis. 2013;17(2):174-8

10. Verani JR, Seitz A, Gilman RH, LaFuente C, Galdos-Cardenas G, Kawai V, et al. Geographic variation in the sensitivity of recombinant antigen-based rapid tests for chronic Trypanosoma cruzi infection. Am J Trop Med Hyg. 2009;80(3):410-15.

11. Paucar R, Moreno-Viguri E, Pérez-Silanes S. Challenges in Chagas disease drug discovery: a review. Curr Med Chem. 2016, 23(28):3154-70.

12. Arias EA, Olivera LV, Bizai ML, Suasnabar S, Arias ED, Fabbro D. A case of chronic Chagas disease: post-treatment serological negativization, is it the same as a cure? Rev Fed Argentina Cardiol. 2017;46(1):Enero-Marzo. Available from: http://www.fac.org.ar/2/ revista/17v46n1/clinicos/01/arias_ingles.php 